\title{
Relationship between left-handedness and increased intelligence among university undergraduates
}

\author{
James Adeniyi Adekoya, Abiodun Adekunle Ogunola \\ Department of Sociology/Psychology, Olabisi Onabanjo University, Ago-Iwoye, Nigeria \\ Email address: \\ jamesadekoya2010@yahoo.com (J. A. Adekoya),psychabiodun@gmail.com (A. A. Ogunola)
}

To cite this article:

James Adeniyi Adekoya, Abiodun Adekunle Ogunola. Relationship between Left-Handedness and Increased Intelligence among University Undergraduates. Psychology and Behavioral Sciences. Vol. 4, No. 2, 2015, pp. 44-50. doi: 10.11648/j.pbs.20150402.12

\begin{abstract}
The focus of the study is to examine the relationship between left-handedness and increased intelligence among university undergraduates of Olabisi Onabanjo University, Ago-Iwoye. Historically, left-handed individuals have had to cope with many difficulties. The level of hand dominance, however, not only depends on the hand with which an individual writes but also on an entire system of processes working together: eye, hand, and foot dominance. Primary data was used for the study. The study sample consisted of both left-handers and right-handers who were drawn from various departments. A structured questionnaire was used to collect data and which were analyzed using appropriate descriptive and inferential statistics. The results showed that male left-handers $(\mathrm{x}=115.50)$ were more intelligent than female left-handers $(\mathrm{x}=101.34)$. The results also showed that left-handers with higher educational level were more intelligent than those with low educational level $(\mathrm{t}=6.93$, $\mathrm{p}<0.05)$. The results also showed that left-handers will be more intelligent in the areas of spatial and musical intelligence than right-handers. The study opined that left-handed individuals can do well in the areas of spatial and musical intelligence and that being a left-hander does possess some advantages for the individual as it would improve his/her retention and memory if well utilized.
\end{abstract}

Keywords: Left-Handedness, Intelligence, Hand Dominance, Retention, Memory, Left-Handers, Right-Handers

\section{Left-Handedness and Intelligence}

Due to the mental abilities that human beings possess, they are unique from other species of creation and this has helped them to discover the facts of the world and to make significantly great progressions in every aspect of scientific achievements and in indeed all human endeavours. Despite these mental capacity that we all share as members of the human community, many differences exist among us which account for the individuality of each person and one of such is the notion of handedness.

In the cerebral cortex of the forebrain, when viewed from the top is divided into two mirror-images which are known as the left and right hemisphere. With the exception of the internal organ such as the heart, the body is basically bilaterally symmetrical, that is the receptive and control centres for one side of the body are in the opposite hemisphere brain. Most times it is said that there's often a tendency, sometimes strong, for one or the other hemisphere to be especially dominant in the different process or functions known as lateralization. Lateralisation is also the preference for using one side of the body more than the other in performing special tasks depending upon which hemisphere is dominant (Rice, 1993; Cardwell, 2003). That is, if the left hemisphere of a person is more dominant than the other, the person tends to be right handed. On the other hand, if a person's right hemisphere is more dominant, the person tends to use the left hand more than the other (usually known as the "left-handers")

A surprise concerning lateralisation in the cerebral cortex involves different specialisation between the two cerebral hemispheres. Despite their mirror-image appearance, the two hemispheres do not mirror one another's abilities. Instead, each hemisphere tends to specialise in different abilities and tend to possess different kind of information. This is to say that some process or functions can happen in only one particular hemisphere. Different functions or actions within the brain tend to rely more heavily on one hemisphere or the other or tend to be performed differently in the two hemispheres. The right hemisphere seems to be somewhat more specialised than the left hemisphere for non-verbal, spatial, and more perceptual information processing and mental rotation of shapes (Ellis 1983). That is, left handers 
are better in these areas.

Left handedness is also more common among musician, mathematician, professional baseball and cricket players, architects and artists (Rice, 1998). Left-handers are also seen to be more creative, more likely to notice the size, shape and form of things, more likely to see the whole picture or concept. All these show that they have more power of perception as compared to right-handers. (McManus, 2002).

Past research has shown that increase in the ratio of lefthanders can produce a corresponding intellectual advances and leap in the number of mathematical, sporting or artistic geniuses. (McManus, 1997). Many researchers in the past have tried to study the brain functioning of intelligent people. L. L. Thurstone $(1938,1955)$ found that he could carve intelligence into seven distinct factors called primary mental abilities - word fluency, verbal, comprehension, spatial ability, perceptual speed, numerical ability, inductive reasoning and memory.

Research further goes to show that the speed with which people are able to retrieve information is related to intelligence. From studies on the brain, it has been discovered that the right hemisphere is more activated during perceptual tasks. Therefore intelligent people may have a more specialised right hemisphere (Barlow, 2001) due to its role in the brain. The length of time a person passed in school influence a person's IQ. Therefore, indirectly education affects a person's level of intelligence (Steinberg, 1990).

For many, school is the first place a child is socialized and therefore will be the first place he/she becomes aware of his/her handedness. Historically teachers have resorted to unthinkable means to make left-handed students become right-handed (Milsom, 1995). Teachers are not the only people that change the preferred hand of the child: parents play a major role in presenting and sticking with the idea of being left-handed as unacceptable.

In the Yoruba culture - the people of South-West Nigeria for instance, it is societally and culturally wring to give something to somebody with the left hand be it an adult or somebody younger to you. It is a mark of total disrespect for the other party if an individual offers something to that party with his left hand. Also, in the culture, the left hand is considered to be used for something dirty, for something of little or no value. For instance the Yoruba man use the left hand to clean himself after defecating, he also uses it to pick rubbish on the ground, to hold something dirty, stinking, or irritating for possible disposal. Hence, they do not strongly fancy the usage of the left hand. At a psychological level, the usage of a left hand for social interactions can sometimes in the Yoruba culture mean that that individual lacks respect, may not do well in life, and might mean a life of lack or dejection. 'Osi' means 'left' and could equally be translated as 'Osi' meaning 'poverty'. Hence, the direct frowning of the usage of the left hand by the Yorubas.

The difficulties faced by left-handed people brings about questions regarding their psychological wellbeing. For some children, the psychological difficulties can be onerous, including feelings of shame, abnormality, and inadequacy.
Difficulty for left-handed individuals can develop early. Toys and clothing tend to have a right-hand bias. Part of children's early development is to learn to adapt and find effective ways of making things work (Milsom, 1995). This raises the question of what happens to those children and adult that are unable to accommodate and what the psychological ramifications are for the left-handed child. Not only are lefthanded people laden with unflattering nicknames, they have also been the subjects of abusive treatment following their natural instincts.

\section{Statement of the Problem}

Most people have heard of these phrase "left brain versus right brain issues", often from the popular press. Such treatments are notorious for exaggerating and over simplifying what is known about laterality and hemispheral specialisation.

There have also been cases where people argue that righthanders are more intelligent than left handers, that is why they would prefer to change their children's handedness. This research is aimed at finding out the truth of such argument.

In Africa, people see the act of using the left hand for doing normal tasks as disrespectful. For instance, in Nigeria, it is seen as an act of disrespect for a person to greet or give another person (especially an adult) something with the left hand. Further making or causing the left hander to learn to use the right hand for most of his activities or tasks which is usually difficult and unfavourable for the lefthander, since it's not where the individual's strength lies.

In addition, most of the machineries and new technological gadgets such as cars, sewing machines, etc, are mostly designed for right-handers which are usually the reverse for left-handers who seem not to be considered in these developments.

In Nigeria most parent, guidance, teachers usually try to change the spatial ones (lefthanders) into right-handers because they feel it is out of culture or a sign of disrespect which in most cases lead to poor handwriting, slow reasoning etc. This create a lot of difficulties for left-handers in our society in terms of their psychological, educational and social functioning.

\section{Theoretical Background}

\subsection{Split Brain Theory (Dr Roger W. Sperry)}

The overall result of Sperry's research can be summarised by his quote: "Everything we have seen indicates that the surgery has left these people with two separating minds. That is, two separate spheres of consciousness".

Some specific differences between the two hemisphere resulted from this and subsequent research. The right brain is better at:

One particular difference in the patient with damage to one side of the brain is how they copy a diagram. A patient with left brain damage will tend to copy the overall outline of a 
diagram but not the details and a patient with right brain damage will tend to get the detail correct but not the overall outline.

Table 1. The activities of each hemisphere of the brain

\begin{tabular}{|c|c|c|}
\hline & Right Hemisphere & Left Hemisphere \\
\hline \multirow[t]{10}{*}{ Specialities } & Copying of design & Language skills \\
\hline & $\begin{array}{l}\text { Discrimination of shapes } \\
\text { e.g. picking out }\end{array}$ & Skilled movement \\
\hline & A camouflaged object & $\begin{array}{l}\text { Analytic time } \\
\text { sequence }\end{array}$ \\
\hline & $\begin{array}{l}\text { Understanding geometric } \\
\text { properties }\end{array}$ & \\
\hline & Reading faces & \\
\hline & Music & \\
\hline & Global holistic processing & \\
\hline & Understanding of metaphor & \\
\hline & Expressing emotions & \\
\hline & Reading emotions & \\
\hline Shared & $\begin{array}{l}\text { Sensation on both side of } \\
\text { face }\end{array}$ & \\
\hline & $\begin{array}{l}\text { Sound perceived by both } \\
\text { ears }\end{array}$ & \\
\hline & Pain & \\
\hline & Hunger & \\
\hline & Position & \\
\hline Emotions & $\begin{array}{l}\text { Negative emotions (fearful } \\
\text { mournful feeling) positive } \\
\text { emotion }\end{array}$ & \\
\hline Neurotransmitters & $\begin{array}{l}\text { Higher level of } \\
\text { Norepinephrine }\end{array}$ & $\begin{array}{l}\text { Higher level of } \\
\text { Dopamine }\end{array}$ \\
\hline
\end{tabular}

\subsection{Brain Dominance Theory}

Ned Herrmann "Father of brain dominance technology" drew on the work of the Sperry and developed the theory brain dominance where people develop a dominant mode of thinking preference. These can range from an analytical "left brain" approach to "right brain" approaches involving pattern matching and intuitive understanding. These preferences have their roots in our genetic makeup and how it affects our underlying cognitive capabilities. For example left-right handed preferences have been observed in the womb. As we developed we tend to respond with our strongest abilities as these lead to quicker short -term reward. This can create a positive feedback system that will strengthen those abilities. Eventually this can lead to a powerful preference for one style over the other and a dislike and discomfort for other mode of thinking.

Herrmann then went onto develop the four-quadrant model of cognitive preference and a questionnaire called the Herrmann Brain Dominance Instrument(HBDI), .The inspiration for this model came from dividing the brain into as four different systems with four preferred style;

A Quadrant: Analytical Thinking.

- Key word: logical, factual, critical, technical and quantitative.

- Preferred activities: collecting data, listening to informational lectures, reading textbooks. Judging ideas based on facts, criteria and logical reasoning

B Quadrant: Sequential Thinking.
- Key word: conservative, structured, organised, detailed and planned.

- Preferred activities: following direction, repetitive detailed homework problems, time management and schedules.

C Quadrant: Interpersonal Thinking.

- Key word: kinaesthetic, emotional, spiritual, sensory, feeling.

- Preferred activities: listening to and sharing ideas, looking for personal meaning, sensory input and group study.

D Quadrant: Imaginative Thinking

- Key word: visual, holistic, intuitive, innovative, and conceptual.

- Preferred activities: looking at the big picture, taking initiative, simulations (what if questions), visual aids. Appreciating beauty of a problem, brain storming.

One of the central ideas of the Hermann's approach is to develop "whole brain thinking". This focuses on strengthening the weak points by using techniques that require a particular style of thinking. This can lead to "creative problem solving" where a combination of different techniques can be used to arrive at a better solution.

\subsection{Review Of Related Studies}

Much of the theories of left-right specialisation has been developed through examining patients who have physical defect in one part of the brain. One of the earliest of these investigations was Paul Broca's work in 1861 which he nicknamed Tan who had a large cyst in the left hand side of the brain. Tan could only one word: "tan", hence the nickname. This indicated that some language function were concentrated in the left side of the brain. Further study of eight patients who all had language problem revealed they also had left hemisphere lesion and the study of the left-right specialisation was born.

Since Broca's early work there has been much research into the processing of language. Several specific areas of the brain have been identified which plays a part in language (for most people these all reside in the left hemisphere).

- Broca's area: Plays a part in the grammatical processing.

- Wernicke's area: Naming object (syntactical processing)

- Angualar gyri: Involved in the recognition of visual symbols

- Supra-marginal gyri:

In most (97\%) right-handed people language is controlled by the left hemisphere. Left-handers have a more even distribution of language in both hemispheres. In $19 \%$ this is concentrated in the left hemisphere, and in $68 \%$ it is concentrated in the right hemisphere, the remainder have language processing in both hemispheres.

Annett \& Manning (1989, 1990a,b) have proposed that left-handedness maintained by a balanced polymorphism whereby the rs $+/$ - heterozygote manifest increase intellectual ability compared with the rs-/- and rs $+/+$ homozygote.

In their research they demonstrated that their method of 
dividing subjects into putative genotype does not allow the rs+/- genotype with handedness groups. Another alternative carried out does not allow heterozygote right handers to be compared both with rs $+/+$ and rs-/- homozygote using their method in undergraduate no evidence was found that suppose heterozygote are relatively more intellectually able than homozygote's on tests of verbal IQ, spatial IQ, diagrammatic IQ or vocabulary.

Theoretical polymorphism hypothesis reveals additional limitations. Although estimation of the size of the heterozygote advantage suggests that it must be very large (21 or 45 IQ points) to explain the effect found by Annett \& Manning, it is nevertheless must be small(3, 4 IQ points) to be compatible with the known differences between right and left handers in social class \& intelligence. Moreover power analysis shows that the latter effect size is too small for Annett \& Manning to have found effect in their studies. Additionally power analyses shows that studies looking for effect in groups of high intellectual abilities, such as university students, are capable of finding significant result, despite Annett claimed such effect. If the Annett \& Manning does demonstrate differences in intellectual ability related to skill. Asymmetry are unlikely to result to balanced polymorphism but instead probably reflect motivational or other differences between right handers of high and low degree of laterality.

Another related study on left-handedness includes Twin theory - This theory postulates that left handed individuals were originally part of an identical twin pair, with the right handed foetus failing to develop early.

Although Australian researcher have debuked the related vanishing twin theory. It is yet unexplained why twin children have a high frequency of left-handedness/righthandedness in pairs.

Another researcher suggested that people with long term impairment of the right hand are more likely to become lefthanded even after the right hand heals.

Some researchers claim that we are more intelligent and eloquent that our right-handed counterparts. In tests conducted by Dr. Alan Searleman from St Lawrence University in New York, he found that left-handers can be considerably more intelligent.

\subsection{Research Hypotheses}

$\mathrm{H}_{1}$ : It was expected that male lefthanders will be more intelligent than female lefthanders.

$\mathrm{H}_{2}$ : It was expected that lefthanders with higher educational level will be more intelligent than those with low educational level.

$\mathrm{H}_{3}$ : It was expected that lefthanders will be more intelligent in areas of spatial and musical abilities than right handers.

\section{Methodology}

\subsection{Research Design}

The design adopted for this research study is survey, designed to collect information / data through the use of questionnaire. This design was chosen basically to access left-handedness and its influence on students' intelligence.

\subsection{Research Instruments}

This study was carried out with the use of questionnaire to gather data. The questionnaire was structured into four different sections namely;

Section A: this consisted of demographic variables such as age , gender, religion ,marital status and levels of students in the university.

Section B: this consisted of scale that measures handedness. Questions in each item are asked for the awareness of hand dominance. The options in the scale are for the participants to choose either (left) or (right).

Section $C$ : this section is the multiple intelligence inventory by Howard Gardner (1983). The scale was designed in alikert scale format, the items used was twenty five. The questions were asked in line with the seven types of intelligence by Howard Gardner which are verbal linguistics, logical - mathematical, visual - spatial, body kinaesthetic, auditory - musical, interpersonal communication, intrapersonal communication.

The likert format goes thus
(5)-Strongly

Disagreed,(1)-Strongly Disagreed.

The higher the score the higher the individuals intelligence, the lower the score the lower the intelligence level.

\subsection{Participants}

In the course of carrying out this study, the following procedures were taken. The total number of pupil used for this research was two hundred students (200). The (200) was divided into two. (100) participants were lefthanders while the remaining hundred were right handers. The questionnaire was distributed to all participants along with the reassurance of confidentiality of their response.

The participants of this study were from various department and levels in the university. Questionnaires was distributed to students in school, place of worship and in their various halls. The whole duration of the field work was a total of five days after which the questionnaires were retrieved. Researcher could only retrieve (170) out of the (200) questionnaire distributed, after which these questionnaires were score and properly analysed using the statistical software called (SPSS)

\section{Results}

\subsection{Socio-Demographic Characteristics of Participants}

The table above presents the distribution of respondents by their socio-demographic characteristics. 108 of the respondents were male indicating $63.5 \%$ while $62(36.5 \%)$ were female. This shows that majority of the respondent that constituted the target population were males. $23(13.5 \%)$ of 
the respondents were below the age of 21 . Respondents between $21-24$ years of age were $86(50.6 \%)$, those between the ages of 25-28 years of age were $59(34.7 \%)$ and those above 28 years of age were $2(1.2 \%) .92(54.1 \%)$ of the respondents were Christian and $68(40 \%)$ were Islam while $10(5.9 \%)$ were Others. This shows that the majority of the respondents were Christian. $11(6.5 \%)$ of the respondents were 100 level students, 28(16.5\%) were 200 level students and $82(48.2 \%)$ were 300 level students while 49(28.8\%) were 400 level student.

Table 2. Socio-demographic characteristics of participants

\begin{tabular}{llll}
\hline Variables & Group & Frequency & Percentage \\
\hline Sex & Male & 108 & 63.5 \\
& Female & 62 & 36.5 \\
Age & Total & 170 & 100.00 \\
& Below 21 years & 23 & 13.5 \\
& 21-24 years & 86 & 50.6 \\
& 25-28years & 59 & 34.7 \\
Religion & Above 28 & 2 & 1.2 \\
& Total & 170 & 100.00 \\
& Christian & 92 & 54.1 \\
Level & Islam & 68 & 40.0 \\
& Others & 10 & 5.9 \\
& Total & 170 & 100.00 \\
& 100 & 11 & 6.5 \\
& 200 & 28 & 16.5 \\
& 300 & 82 & 48.2 \\
& 400 & 49 & 28.8 \\
& Total & 170 & 100.00 \\
\hline
\end{tabular}

\subsection{Hypotheses Testing}

Hypothesis one: it was expected that male lefthanders will be more intelligent than female lefthanders.

Table 3. The intelligence of male and female lefthanders

\begin{tabular}{lllllll}
\hline Sex & N & Mean & STD & t-cal & P & Remark \\
\hline Male & 79 & 115.50 & 5.09 & 5.80 & $<0.05$ & Significant \\
Female & 26 & 101.34 & 19.96 & & & \\
\hline
\end{tabular}

The table above presents an independent sample t- test showing whether male lefthanders will be more intelligent than females' lefthanders or not. The result of the descriptive analysis presented above states that the mean score for male lefthanders is 115.50 while that of female is 101.34 with standard deviation of 5.09 and 19.96 respectively. The mean deviation difference was at both $5 \%$ and $10 \%$ level. Thus male lefthanders will be more intelligent than female lefthanders.

Hypothesis Two: it is expected that lefthanders with higher educational level will be more intelligent than those with low educational level.

Table 4. Level of Intelligence based on education of lefthanders

\begin{tabular}{lllllll}
\hline Educational level & $\mathbf{N}$ & Mean & STD & t-cal & P & remark \\
\hline High & 84 & 114.34 & 7.59 & 4.41 & $<0.05$ & Significant \\
Low & 20 & & & & & \\
\hline
\end{tabular}

The table presents an independent sample t- test showing whether left handers with higher educational level will be more intelligent than those with low educational level or not. The result of the descriptive analysis presented above shows the mean intelligent score for the lefthanders with higher educational level is 114.35 while that of low educational level is 101.80 with standard deviation of 7.59 and 21.21 respectively. The mean difference was significant at both $5 \%$ and $10 \%$ level. Thus, lefthanders with higher educational level would be more intelligent than those with low educational level. Nevertheless, the t-value of 4.41 whose probability close to zero percent shows statically that at $5 \%$ and $10 \%$ level of significant, there is enough evidence to conclude that lefthanders with higher educational level will be more intelligent than those with low educational level.

Hypothesis Three: it was expected that lefthanders will be more intelligent in areas of spatial and musical than right handers.

Table 5. Intelligence in special and musical abilities

\begin{tabular}{lllllll}
\hline Group & N & Mean & STD & t-cal & P & Remark \\
\hline Right-handers & 64 & 97.46 & 14.23 & 6.93 & $<0.05$ & Significant \\
lefthanders & 104 & 111.94 & 12.42 & & & \\
\hline
\end{tabular}

The table above present an independent sample t- test showing whether lefthanders will be more intelligent in areas of spatial and musical abilities than right-handers or not.

The result of the descriptive analysis presented above shows that the mean intelligence score for right-handers students in the areas of spatial and musical abilities is 97.46 while that of lefthander is 111.94 with standard deviation of 14.23 and 12.42 respectively. the mean difference were significant at both $5 \%$ and $10 \%$ level. Thus, lefthanders will be more intelligent in areas of spatial and musical ability than right-handers.

Nevertheless, the $\mathrm{t}$ value of 6.93 whose probability close to zero percent shows statistically that at $5 \%$ and $10 \%$ level of significant, there is enough evidence to conclude that lefthanders will be more intelligent in areas of spatial and musical abilities than right handers.

\section{Discussion of Findings}

It is a widespread syndrome for left-handers to be viewed as people of lesser importance in the society. Still, others view them as nothing more than ordinary. This research serves as an eye opener into the correlation between handedness and intelligence.

The first hypothesis stated that it was expected that male left-handers will be more intelligent than female left-handers. The result gives reasons to believe that this is true as the analysis shows that it is significant. The result from the data gathered showed that more of the male heft-handers, about $35 \%$, have abilities that the females don't have when it comes to activities like working with computers and calculators, mathematics, drawing and map reading sports, musical instruments, etc.

The second hypothesis stated that individuals with higher educational level were expected to perform better than those 
with lower educational level. In Steinberg's findings, he stated that IQ is influenced by the length of time a person passed through school and so is indirectly means that education affects the level of intelligence even among lefthanders. Further on this, Murphy observed that most IQ differences that are of concern are those that are well within the normal range and might be systematically modified by optimal structuring of the learning environment. In view of the foregoing, it could be concluded safely that the educational level of individuals really does have a part to play when it comes to their performance.

The third hypothesis stated that it is expected that lefthanders will be more intelligent in areas of spatial and musical abilities than right-handers. This, in fact is what the result of the information gathered by the researchers pointed out. To put this into perspective, $45 \%$ of the lefthanders claim to be more intelligent in these areas. Diana Deutcsh (1978) reported that people who are left-handed and display more heterogeneity in terms of both direction and degree of cerebral dominance.

\section{Conclusion}

This study examined left handedness and increase intelligence among OOU students. This objective is achieved by determining if male left-handers are more intelligent than female left-handers. Consideration was also given to the educational level of the individuals and their ability to perform well in areas of spatial and musical abilities composed to right-handers.

From results gathered, it was obvious that while some other factors might contribute to the intelligence level of an individual, left handedness offers an additional advantage over right handedness. This can be said to be that left-handed individuals will be more intelligent in the areas of spatial and musical intelligence because they would have a large repository of control because this area is controlled by the hemisphere responsible for their left-handedness.

Also, educational level and environment has a link with the intelligence of the individual. In fact, the IQ of an individual can be improved by training. That is, a left-handed individual can achieve giant strides academically because the asymmetry of the brain hemispheres gives him/her good coordination, retention, and memory.

\section{Recommendation}

The government should assist in bringing out their potentials by building institutions that can help them develop in different aspects of learning including creative arts, sciences, athletics, music and so on since they are likely to excel in these areas. The government should also make resources available to researchers for more meaningful findings and better understanding of this wonderful phenomenon.

Parents are the closest to their children especially in their early years. They need to be sensitized as to the uniqueness of left-handedness and helped to appreciate it. They are to desist from trying to change their handedness from left to right simply on the basis of societal rejection of lefthandedness.

New tools have been designed for the lefthanders, to make things easier for them and it is necessary for the parents and teachers to take advantage of these tools e.g scissors, pen that are majorly designed for the

Lastly, it is necessary that teachers especially those in nursery and primary schools be educated on left-handedness because they are usually the ones that spend most time with young children and it as been observed that these teachers mostly punish the lefthanders unjustly when they use the lefthand for writing or eating, which is wrong on the part of the teachers.

So, these teachers should be properly educated on this, so they do not turn the lefthanders into poorly impaired individuals as a result of punishment and force applied on these ones to change their handedness.

\section{References}

[1] Annet \& Marrian (1993) Annulet's Theory That Individuals Heterozygous for the Right Shift Gene Are Intellectually Advantageous. British Journal of Psychology, 84, 539 - 544.

[2] Annet, M \& Manning, M (1990) Reading and a Balanced Polymorphism for Laterality and Ability. British Journal of Child Psychology and Psychiatry, 31 (4), 514.

[3] Barlow, H. D. (2001) Casebook in Abnormal Psychology (2nd Ed.) New York: Wadsworth Thomson Learning.

[4] C. G. Jung, Gerhard Adler, R. F. C. Hull, Psychological Types (Collected Works of C. G. Jung Vol. 6) Bollingen, 1976.

[5] Cardwell, M. (2003) Complete A - Z Psychology Handbook. (3rd Ed.) London. Hodder and Slottghoton.

[6] Cecci, S. J. (1990) \{On Intelligence ... More Or Less: A BioEcological Treatise On Intellectual Development Englewood Cliffs, N. J.: Prentice-Hall.

[7] Cole, M. (1997) Reading on the Development of Children (2nd Ed.) New York: W. H. Freeman And Company.

[8] David Lazear, Eight Ways of Knowing. Teaching for Multiple Intelligence (3rd Edition). Skylight Professional Development, Illinois, 1999.

[9] Deutsch, D. (1998). Centre For Human Information And Processing, University Of California.

[10] Gardener, Frames Of Mind: The Theory of Multiple Intelligence, (1993) ISBN 0465025102. (1993 Ed.)

[11] Hunt, E. (1997) Handbook of Perception and Cognition (2nd Ed.). San Diego: Academic Press.

[12] McManus, C (2002, March 2) Research into Left-Handedness and Its Effects. Time, Retrieved December 13, 2004, from http://www.geocities.com

[13] Milsom, L. (1995). Left handed children are they losing out? Educational Media International, 32, 107-108. 
[14] Murphy, R. K. (1998) Psychological Testing (4th Ed.) New York: Prentice Hall International Inc.

[15] Myers, G. D. (2001) Psychology (6th Ed.) New York: Worth Publishers.

[16] Ned Hermann, the Creative Brain, Brain Books, Lake Lure, North Carolina, 1990.

[17] Needlemann, R. (2001, June 10) Left-handed Thinking. Times. Retrieved June 7, 2005 from http://www.psychology/handedness/lefthandedthinking.htm
[18] Morris, R. J. (2006) Left Brain, Right Brain, Whole Brain? An Examination into the Theory of Brain Lateralization.

[19] Rice, P. F. (1998) Human Development (3rd Ed.) New Jersey: Prentice Hall.

[20] Saba Ghayas and Adnan (2007)Adil Journal of the Indian Academy of Applied Psychology. January 2007, Vol. 33, No. $1,85-91$.

[21] Steinberg (1990) Adolescence. New York: McGraw Hill Inc. 